\title{
Yersinia pseudotuberculosis infection as a cause of reactive arthritis as seen in a genitourinary clinic: case
} report

\author{
R I LINDLEY,* R S PATTMAN, $†$ M H SNOW $\ddagger$ \\ From the *Department of Haematology, Royal Victoria Infirmary, and the Departments of †Genitourinary \\ Medicine and $\ddagger$ Medicine, Newcastle General Hospital, Newcastle Upon Tyne
}

SUMMARY Patients are often referred to the genitourinary clinic for screening for urethral infections after a clinical diagnosis of Reiter's syndrome or reactive arthritis. We report a case of reactive polyarthritis in which serological evidence of Yersinia pseudotuberculosis infection was found in the absence of the other common precipitating organisms, such as Chlamydia spp, Klebsiella spp, Shigella spp, Campylobacter spp, or Yersinia enterocolitica.

\section{Case report}

A man aged 26 presented to the casualty department of Newcastle General Hospital with a history of rigors and a painful swollen left ankle on which he was unable to bear weight. Ten days previously he had developed malaise, shivering, diarrhoea, and vomiting. These symptoms resolved in the three days before his admission to hospital.

On examination he had a temperature of $38.2^{\circ} \mathrm{C}$ and a hot, tender, swollen left lateral malleolus. His white cell count was $11.7 \times 10^{9} / 1$ and the erythrocyte sedimentation rate was $30 \mathrm{~mm}$ in the first hour. Coagulase negative Staphylococcus spp was grown in one blood culture bottle. Although he received intravenous flucloxacillin and benzylpenicillin as a precaution against septic arthritis, this organism was probably a contaminant. He was treated with ibuprofen for analgesia, and his symptoms remitted.

After a month he was readmitted with left ankle and bilateral wrist pain and swelling, and a painful left knee with effusion. He was referred to the genitourinary clinic for investigation of the aetiological cause of suspected reactive arthritis. He had not been abroad in the past year, and his only sexual partner had been his wife. He had no urethral discharge, no balanitis, and a negative result in the two glass urine test. No mucous membrane abnormalities or evidence

Address for reprints: Dr R S Pattman, Ward 34, Department of Genitourinary Medicine, Newcastle General Hospital, Westgate Road, Newcastle Upon Tyne NE4 6BT

Accepted for publication 21 March 1989 of conjunctivitis were seen. He received ibuprofen, and his joints were splinted.

Further investigations showed HLA types A1, A28, B27, and B37, Yersinia pseudotuberculosis type III at a titre of $1 / 160$ (tested at a Public Health Laboratory Service reference laboratory), a white cell count of $9 \cdot 7$ $\times 10^{9} / 1$, haemoglobin concentration of $129 \mathrm{~g} / \mathrm{l}$, and an erythrocyte sedimentation rate of $46 \mathrm{~mm}$ in the first hour. His serum antistreptolysin $O$ concentration was $166 \mathrm{units} / \mathrm{ml}$. He had no serological evidence of rheumatoid factor or infection with Brucella spp, Salmonella spp, Treponema pallidum, or any viruses. Radiography of the sacroiliac joints showed no abnormality. Culture of a urethral smear for Chlamydia trachomatis gave a negative result.

When the results of the serological test for $Y$ pseudotuberculosis became available the patient was given doxycycline $100 \mathrm{mg}$ a day for a month. During the next six weeks his arthritis slowly remitted, although he developed bilateral calcaneal spurs. His erythrocyte sedimentation rate remained between 24 and $40 \mathrm{~mm}$ in the first hour, and six months after presentation he had no detectable antibodies to $Y$ pseudotuberculosis.

\section{Discussion}

$Y$ pseudotuberculosis most commonly presents clinically as a pseudoappendicitis (terminal ileitis, appendicitis, or mesenteric adenitis) ${ }^{12}$ or diarrhoea. An outbreak of $Y$ pseudotuberculosis infections with reactive arthritis has been reported from Turku, Finland, ${ }^{2}$ and similar cases have been seen in Rumania, ${ }^{3}$ but no report from the United Kingdom has been published. 
Increasing numbers of $Y$ pseudotuberculosis infections are being reported in England, Wales, and Ireland (Public Health Laboratory Service, Communicable Disease Surveillance Centre, unpublished observation). In $1985 Y$ pseudotuberculosis infection was reported in 19 patients, four of whom had associated arthritis, in 1986 the figures were 44 (three with arthritis), and in 198794 (nine with arthritis). The reported clinical features associated with evidence of $Y$ pseudotuberculosis infection included erythema nodosum, diarrhoea, abdominal pain, bowel perforation, mesenteric adenitis, pneumonia, suspected Crohn's disease, ileitis, pyrexia, suspected appendicitis, pyelonephritis, inflammatory bowel disease, pancreatitis, "liver lesions", arthralgia, and polyarthritis.
We think that an increased awareness of the association between seronegative polyarthritis and $Y$ pseudotuberculosis infection is important, and we emphasise the importance of considering a large differential diagnosis in any patient presenting with Reiter's syndrome or reactive arthritis.

\section{References}

1 Randall KJ, Mair NS. Family outbreak of Pasteurella pseudotuberculosis infection. Lancet 1962; i:1042-3.

2 Tertti R, Granfors K, Lehtonen O-P, et al. An outbreak of Yersinia pseudotuberculosis infection. J Infect Dis 1984;149: 245-50.

3 Georgescu C, Dan E, Cojocaru M, Plosceanu L. The role of infections with Yersinia pseudotuberculosis and Yersinia enterocolitica in the pathogenesis of chronic inflammatory, rheumatic and collagen diseases. Med Interne 1986;24:217-20. 